\title{
AN APPROACH TO STRUCTURAL HEALTH MONITORING OF COMPOSITE STRUCTURES BASED ON EMBEDDED PZT TRANSDUCERS
}

\author{
Michał Dziendzikowski ${ }^{1}$ \\ Krzysztof Dragan ${ }^{1}$ \\ Artur Kurnyta ${ }^{1}$ \\ Lukasz Kornas \\ Adam Latoszek ${ }^{1}$ \\ Magdalena Zabłocka ${ }^{1}$ \\ Sylwester Kłysz ${ }^{1,2}$ \\ Andrzej Leski ${ }^{1}$ \\ Marek Chalimoniuk ${ }^{1}$ \\ Janusz Giewoń ${ }^{1}$ \\ ${ }^{1}$ Air Force Institute of Technology, ul. Ks. Boleslawa 6, 01-494 Warsaw, Poland \\ ${ }^{2}$ University of Warmia and Mazury, ul. Oczapowskiego 2, 10-719 Olsztyn, Poland
}

\begin{abstract}
One approach to developing a system of continues automated monitoring of structural health is to use elastic waves excited in a given medium by a piezoelectric transducers network. Depending on their source and the geometry of the structure under consideration elastic waves can propagate over a significant distance. They are also sensitive to local structure discontinuities and deformations providing a tool for detecting local damage in large aerospace structures. This paper investigates the issue of Barely Visible Impact Damages (BVIDs) detection in composite materials. The model description and the results of impact tests verifying damage detection capabilities of the proposed signal characteristics are presented in the paper.
\end{abstract}

Keywords: structural health monitoring, PZT sensor network, barely visible impact damages

\section{INTRODUCTION}

Conventional nondestructive testing techniques are nowadays supposed to be complemented by systems of integrated sensors continuously monitoring the health of a structure. The application of such methods would definitely increase safety, especially when considering hardy accessible 'hotspots'. In addition, it could save up to $50 \%$ of time required for inspections depending on the type of an aircraft [1]. Monitoring of composite structures is one of the most important branches of Structural Health Monitoring (SHM) technology. Composites are vulnerable to random impact damages that might occur in the time between the two subsequent inspections, which poses an additional risk for the structure. The wear-out process of composites remains, to a high extent, unknown, at least compared to metallic structures, adversely affecting their safety. Furthermore, even impacts of low energy can form an extensive network of cracks and de-laminations under the surface of an element (Fig. 1) causing severe loss to its strength. The fact that such damages are either invisible or barely visible on the boundaries hinders the effective use of low-cost NDI methods, making a reliable SHM system allowing for automated detection of such damages highly desired by the aerospace industry. 


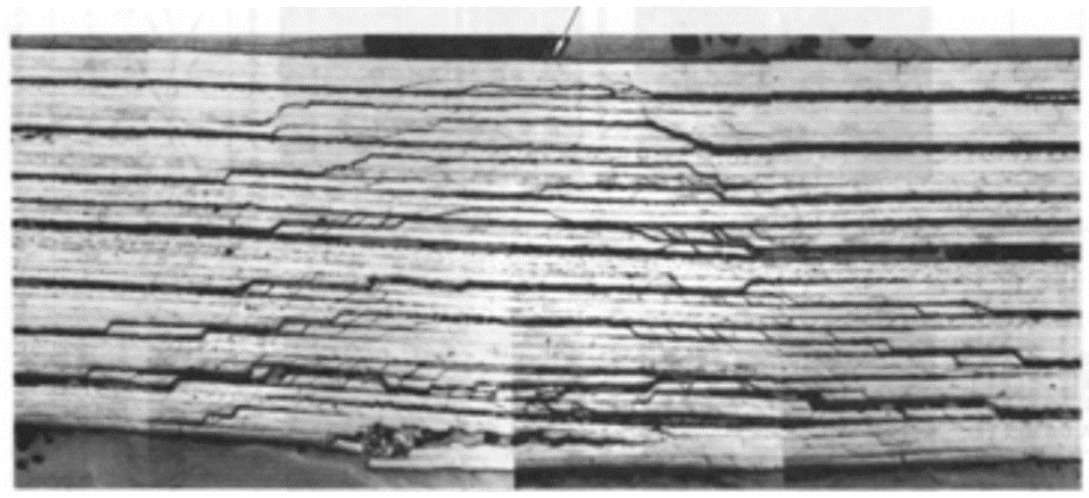

Fig. 1. BVID in CFRP composite structure [2]

\section{HEALTH ASSESSMENT OF COMPOSITE STRUCTURES}

One idea for structural health monitoring systems is based on measuring of the mechanical properties of materials used in an aircraft's structural elements. This approach is based on the analysis of small displacements propagation excited in the element by a network of PZT piezoelectric actuators $[3,4]$. A solution to small deformation dynamics of the medium strongly depends on the boundary conditions, in particular the geometry of the object and its distortions caused by discontinuities and deformations. Structural damages can thus result in observable changes of the signal generated by the network sensors. The state of a monitored structure is assessed based on chosen signal's characteristics called Damage Indices (DIs). The acquired signals can be also influenced by factors other than damages, which poses the risk of false indications. Therefore it is important that the DIs used for the structure assessment be balanced between sensitivity to damages and stability under varying working conditions of transducers. In the adopted approach, the DIs carry the marginal signal information content. Denoting as $f_{g s}^{e n v}$ the envelope of a signal generated by the transducer $g$ and received by the sensor $\mathrm{s}$ and as $f_{g s, b}^{e n v}$ the envelope of the corresponding baseline, i.e. the reference signal obtained for the initial state of the structure, the proposed Damage Indices are given as follows [5]:

$$
D I_{1}(g, s)=1-\operatorname{cor}\left(f_{g s}^{e n v}, f_{g s, b}^{e n v}\right) \quad, \quad D I_{2}(g, s)=\left|\frac{\int\left(f_{g s}^{e n v}-f_{g s, b}^{e n v}\right)^{2} d t}{\int\left(f_{g s, b}^{e n v}\right)^{2} d t}\right|
$$

where $\operatorname{cor}\left(f_{g s}^{e n v}, f_{g s, b}^{e n v}\right)$ stands for the sample correlation of $f_{g s}^{e n v}, f_{g s, b}^{e n v}$. Both of the proposed DIs are correlated not only with the total energy received by a given sensor but also with its distribution in time during the measurement. Structural discontinuities caused by BVIDs dissipate the wave energy due to the wave scatterings on the de-laminations but can also alter its time redistribution due to local stiffness changes and the related propagation speed shift of the incoming wave packets. Both of the effects can be captured by the proposed Damage Indices. As will be shown below, the above DIs change the most whenever a damage occurs on a direct path of the wave propagation between the generator $g$ and sensor s, i.e. the sensing path. In order to decrease further the dependence of DIs on the damage location the Averaged Damage Indices (ADIs) can be used [5]:

$$
A D I_{j}:=\frac{1}{n(n-1)} \sum_{\substack{g, s: \\ g \neq s}} D I_{j}(g, s), \quad j=1,2
$$


where is the number of transducers in a network measurement node. In this way the joint information from all of the sensing paths of the network is utilized in a single Damage Index. Averaged Damage Indices (ADIs) are better suited for damage size estimation and retain the capacity for structural evaluation also in the case of improper functioning of several transducers of the network.

\section{IMPACT TEST}

In order to test BVID's damage detection capabilities in the presented approach, the impact tests of the CFRP (Carbon Fiber Reinforced Plastic) specimens with an assembled PZT sensor network were performed. Impacts with energies: 9J, 6J and 3J were carried out subsequently and after each of them a series of signals generated by the PZT network were collected. The following figures present the subsequent impact localizations (Fig. 2 - Fig. 5) as well as the change of the proposed Damage Indices (DIs) on all of the network sensing paths.
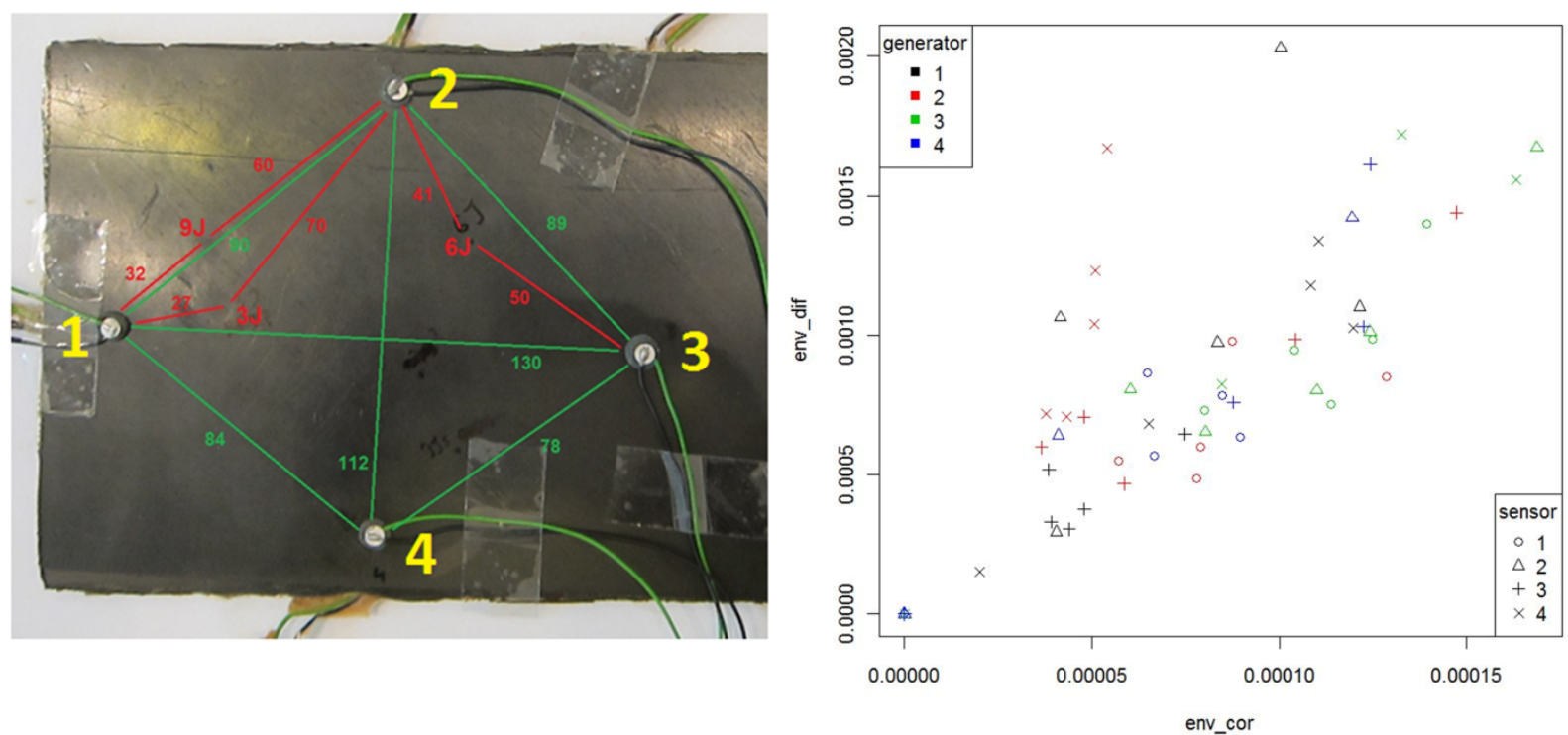

Fig. 2. Damage Indices in the pristine state of the structure
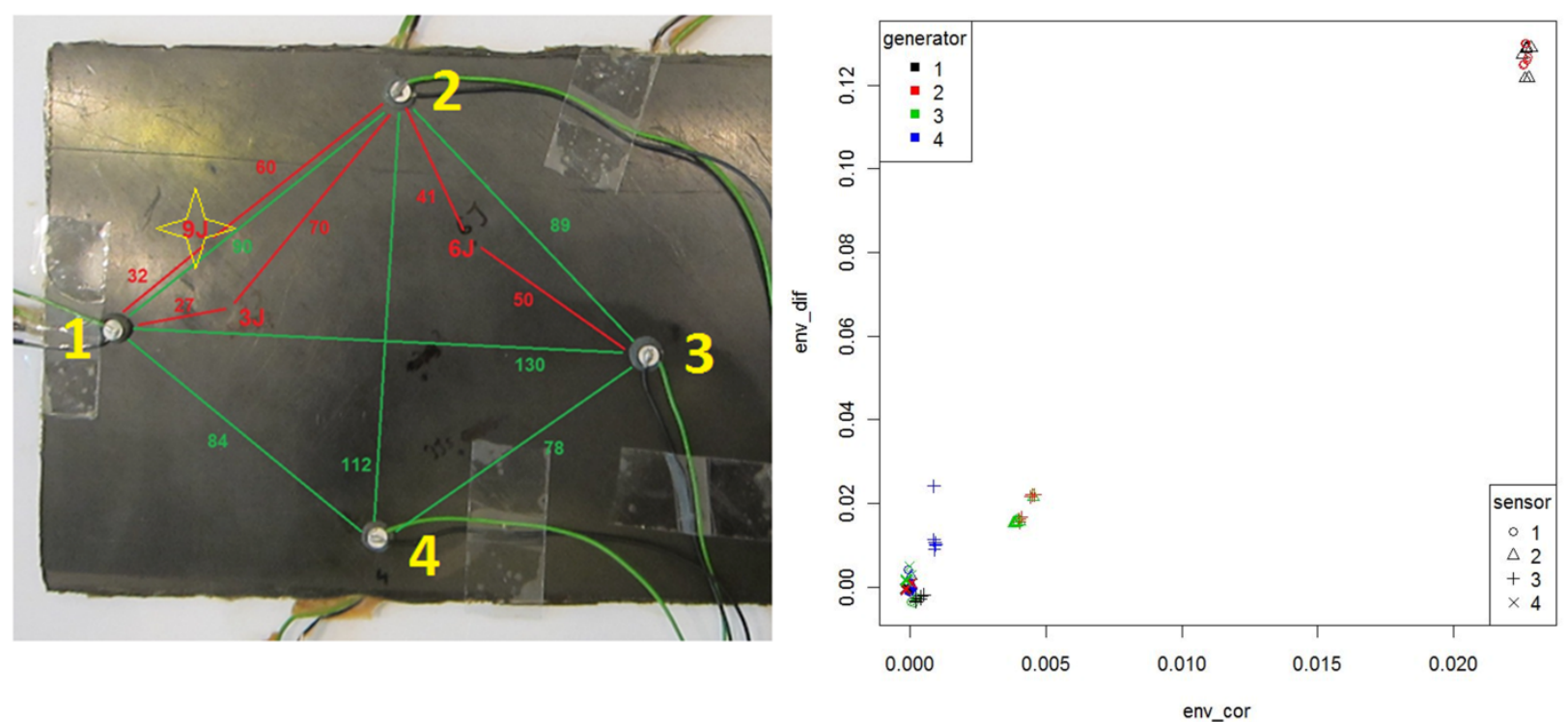

Fig. 3. Damage Indices after 9J impact 

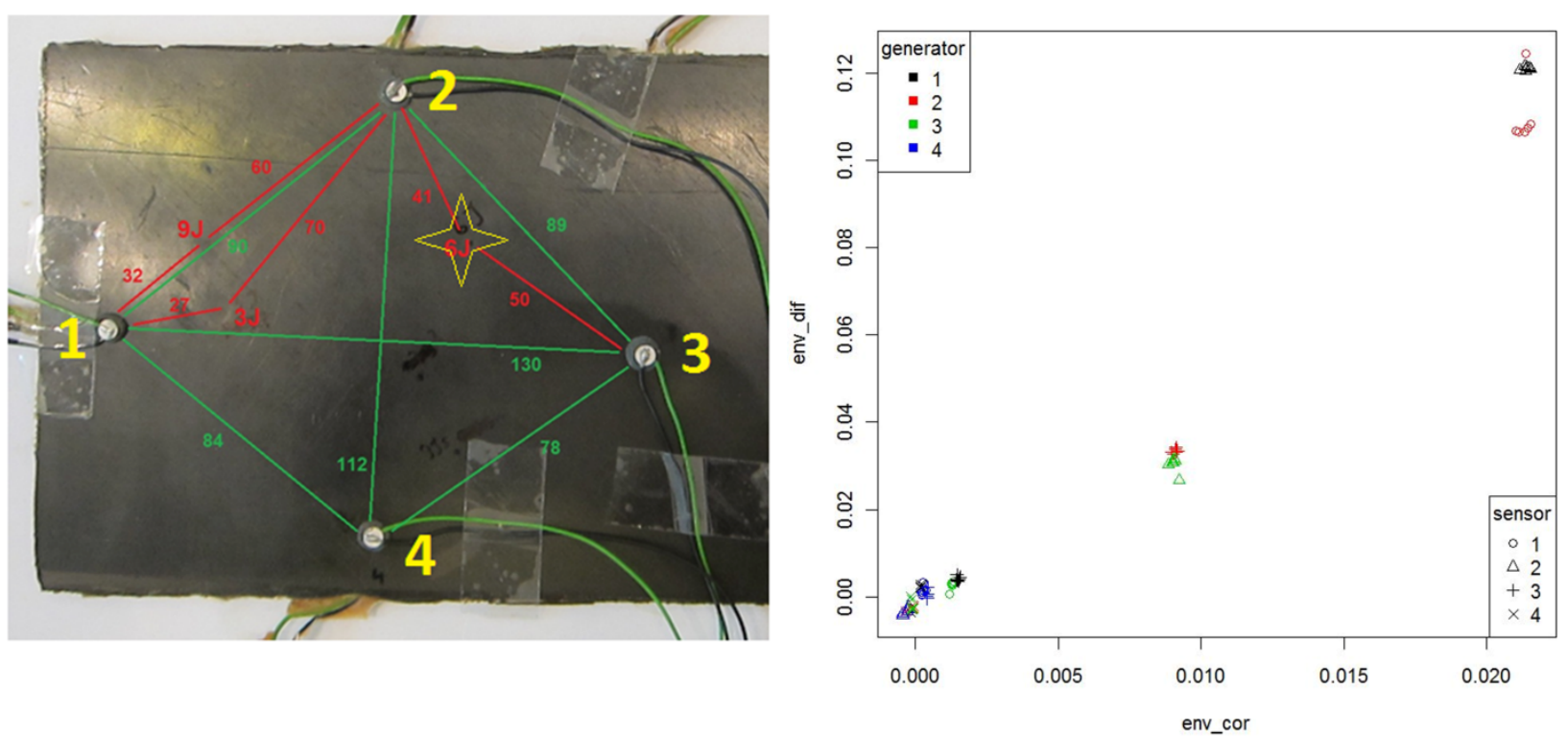

Fig. 4. Damage Indices after $6 \mathrm{~J}$ impact
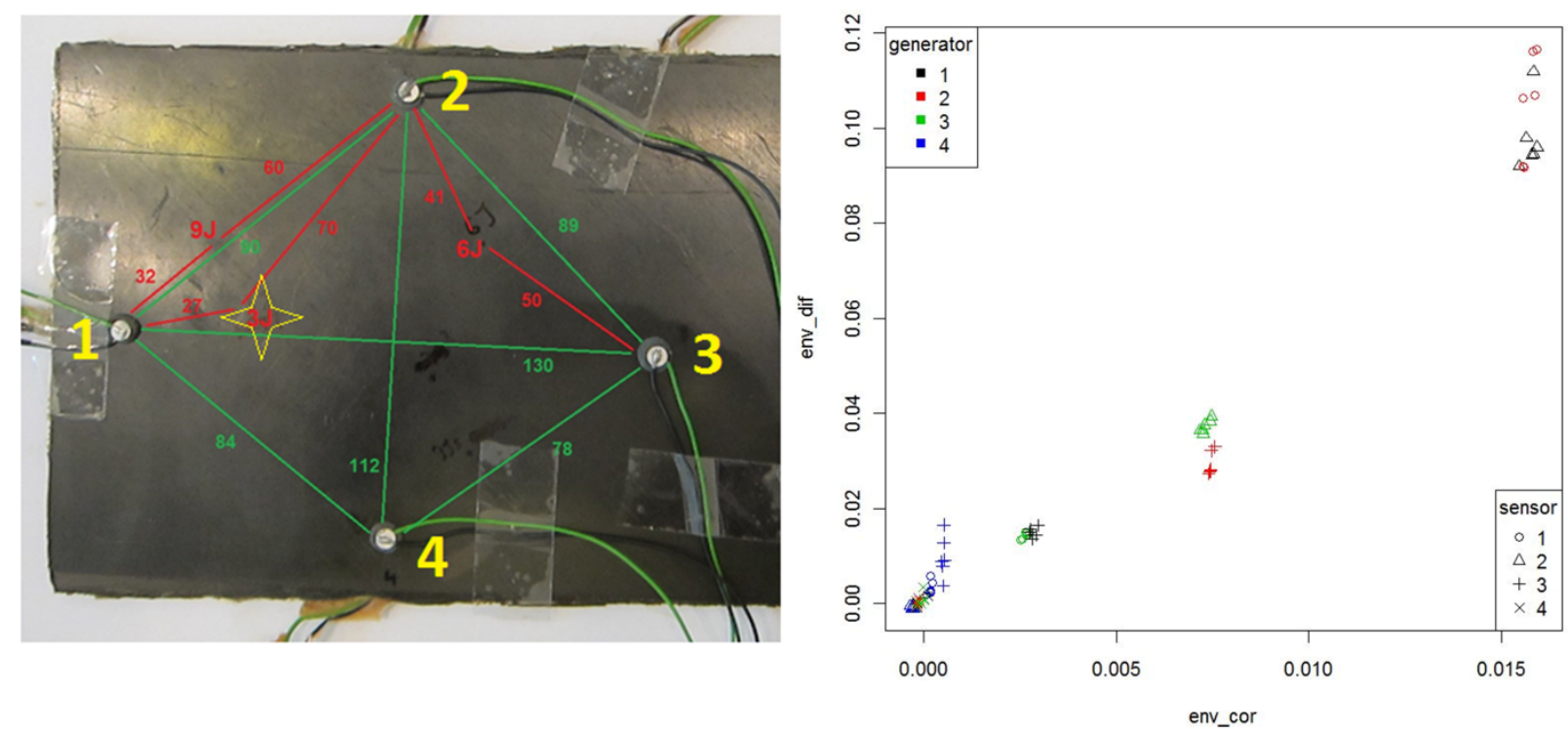

Fig. 5. Damage Indices after 3J impact

The colors of data on the plot correspond to the generator number, whereas their shape codes the information about the sensor. It is evident that the changes of Damage Indices are the effect of the BVIDs. For each impact the change in the DIs occurs only for the sensing path in the proximity of the resultant damage. The value of this change depends on the impact energy and is thus correlated with the size of BVID damage. The plot (Fig. 6) shows the Averaged Damage Indices with respect to the cumulative energy of the subsequent impacts. Both the data corresponding to $9 \mathrm{~J}$ impact and the $6 \mathrm{~J}$ impact are well separated from the base state of the structure as well as from each other. The impact of energy $3 \mathrm{~J}$ results in a slight but significant separation of ADIs, which provides a hint about damage detection capabilities of the system. 


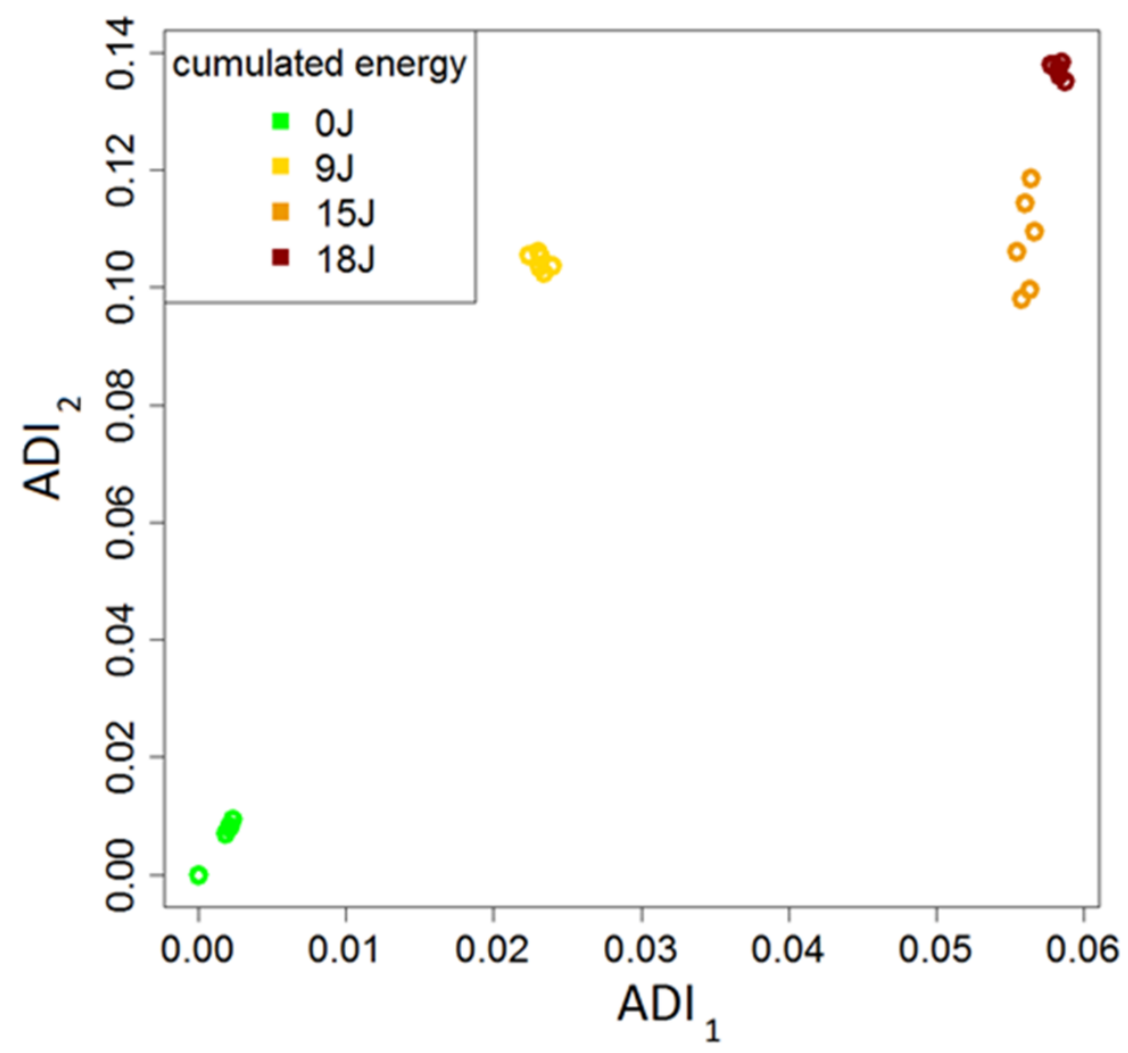

Fig. 6. Averaged Damage Indices

\section{SUMMARY}

Introducing Structural Health Monitoring systems as a compound of the future aircraft structural life management programs seems to be inevitable. In the article, the approach to structure monitoring based on the PZT sensor network and its application to detect barely visible impact damages of composite structures was presented. The technique allows for the detection of impacts with energy higher than 3J. For structures composed of many layers equipped with the transducers separated by media of distinct acoustic impedance, it opens an opportunity to differentiate the damage severity across the layers. However, a lot of effort needs to be made before the system enters operational phase including long term ADI's variable environmental tests as well as POD studies and system certification.

\section{ACKNOWLEDGMENTS}

This work has been partially supported with the European Union funds by the European Social Fund within the project "Rozwój nauki - rozwojem regionu - stypendia i wsparcie towarzyszące dla mazowieckich doktorantów".

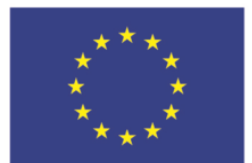




\section{REFERENCES}

1. C. Boller and W.J Staszewski. Aircraft structural health and usage monitoring. In W.J. Staszewski, C. Boller, and Tomlinson G.R., editors, Health Monitoring of Aerospace Structures, pages 29-73. John Wiley and Sons, Ltd, 2004.

2. L. Ilcewicz, CS\&TA Federal Aviation Administration, 2006.

3. V. Giurgiutiu. Structural health monitoring: with piezoelectric wafer active sensors. Academic Press, 2007.

4. Z. Su and L. Ye. Identification of damage using lamb waves: from fundamentals to applications. Springer, 2009.

5. K. Dragan, M. Dziendzikowski, S. Klimaszewski, S. Klysz, and A. Kurnyta. Energy correlated damage indices in fatigue crack extent quantification. Key Engineering Materials, 569:1186-1193, 2013. 